\title{
可调可重构的环形振荡器物理不可克隆函数
}

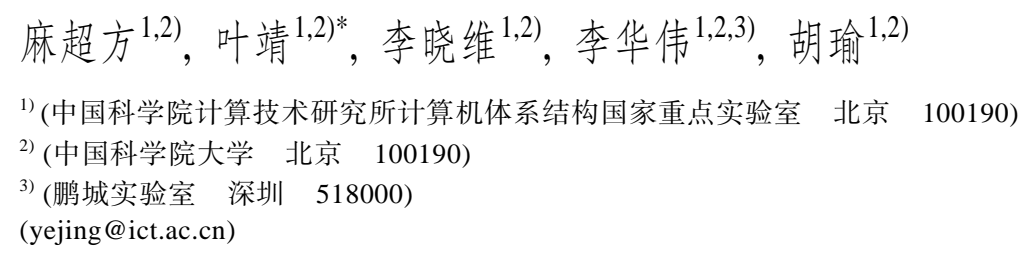

\begin{abstract}
摘 要: 针对环形振荡器物理不可克隆函数均匀性与独特性不够理想的问题, 提出一种可调可重构的环形振荡器物 理不可克隆函数设计. 该设计包含可重构环形振荡器模块、整合器模块和裁决器模块. 可重构环形振荡器模块由多个 独立且具有相同设计的可重构环形振荡器-计数器组构成, 芯片各部分的工艺偏差由计数器的数值反映; 整合器模块 通过将多个计数器数值进行排序并依次作差, 以减弱芯片固有时延对物理不可克隆函数均匀性与独特性的不良影响; 裁决器模块经预先设计阈值后, 对整合器模块产生的差值进行裁决, 产生最终响应. 在 Xilinx Zynq-7000 Soc ZC702 FPGA 实现上述可调可重构的环形振荡器物理不可克隆函数, 实验结果表明, 均匀性的平均值为 $49.36 \%$, 独特性的 平均值为 $47.07 \%$, 均趋于 $50 \%$ 的理想值, 取得了令人满意的效果.
\end{abstract}

关键词: 物理不可克隆函数; 均匀性; 独特性; 整合器; 裁决器

中图法分类号: TP492 DOI: 10.3724/SP.J.1089.2021.18561

\section{Adjustable and Configurable Ring Oscillator Physical Unclonable Function}

\author{
Ma Chaofang ${ }^{1,2)}$, Ye Jing ${ }^{1,2)^{*}}$, Li Xiaowei ${ }^{1,2)}$, Li Huawei ${ }^{1,2,3)}$, and Hu Yu ${ }^{1,2)}$ \\ 1) (State Key Laboratory of Computer Architecture, Institute of Computing Technology, Chinese Academy of Sciences, Beijing 100190) \\ 2) (University of Chinese Academy of Sciences, Beijing 100190) \\ 3) (Peng Cheng Laboratory, Shenzhen 518000)
}

\begin{abstract}
To cope with the problem that the uniformity and uniqueness of the ring oscillator based physical unclonable function are not ideal, this paper proposed a design of the adjustable and configurable ring oscillator physical unclonable function. The design is composed of configurable ring oscillator module, integration module and judgement module. The configurable ring oscillator module is composed of several independent configurable ring oscillator and counter groups which have the same design. The technological deviation of each part of the chip is reflected by the counter value. In order to reduce the undesirable influence of chip intrinsic delay on the uniformity and uniqueness of physical unclonable function, the integration module arranges and differentiates counter values. The judgment module judges the difference of the integration module by designing the threshold value in advance, and produces the final response. The experimental results show that the proposed design implemented on Xilinx Zynq-7000 Soc ZC702 FPGA has an average uniformity of $49.36 \%$ and an average uniqueness of $47.07 \%$, both of which tend to the ideal value of $50 \%$, achieving the expected purpose.
\end{abstract}

收稿日期：2020-08-07; 修回日期：2020-09-29. 基金项目：国家自然科学基金(61704174，61532017，61432017，61521092). 麻超方 (1998一), 男, 硕士研究生, 主要研究方向为硬件安全; 叶靖(1988一), 男, 博士, 副研究员, 硕士生导师, CCF 会员, 论文通讯作者, 主要研究方向为硬件安全、集成电路测试与诊断; 李晓维(1964一), 男, 博士, 研究员, 博士生导师, CCF 会员, 主要研究方向为集成 电路测试与诊断、验证、可靠性设计、无线传感网络; 李华伟(1974一), 女, 博士, 研究员, 博士生导师, CCF 高级会员, 主要研究方 向为 VLSI 测试、验证和可靠性设计; 胡瑜(1975一), 女, 博士, 研究员, 博士生导师, CCF 会员, 主要研究方向为集成电路测试与诊 断、验证、可靠性设计. 
Key words: physical unclonable function; uniformity; uniqueness; integration module; judgment module

随着互联网信息化技术的快速发展，日常生 活中智能电子设备的普及, 在极大地提升人们生 活质量的同时，带来了泄露隐私信息的风险，这向 信息安全技术提出了挑战.

传统的密码学对上述问题的解决方法需要依 赖存储在非易失性存储器 (non-volatile memory, NVM)中的二进制密钥才能实现，一旦二进制密钥 泄露，系统便毫无安全性可言. 近期的研究发现, 攻击者通过多种物理方式可以顺利地窃取存储在 NVM 中的二进制密钥, 从而窃取设备中存储的重 要信息 ${ }^{[1]}$.

\section{1 研究背景}

\subsection{PUF 简介}

物理不可克隆函数 (physical unclonable function, PUF)是一种新型硬件安全原语, 其利用了电 路在生产制造过程中不可避免的工艺偏差产生电 路的独特标识. 通过向电路输人一个激励, 便可以 获取一个由工艺偏差决定的响应(response, R). 这 样的激励与其相对应的响应称为激励 - 响应对 (challenge-response pair, CRP). 由于电路在制造过 程中的工艺偏差难以预测与控制，即使对于相同 设计的电路, 其工艺偏差往往也不相同, PUF 的 CRP 因此也不会相同. 同时, 与传统的在 NVM 中 存储二进制密钥的方式相比, PUF 具有上电可读 取, 掉电即失的特性, 大大提升了其安全性. 这些 特性使 PUF 具有广阔的发展前景, 在防伪认证 ${ }^{[2]}$ 、

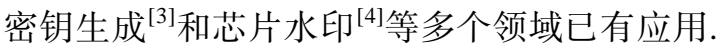

根据 PUF 所具有的 CRP 数量的多少, 可以将 PUF 分类为弱 PUF 和强 $\mathrm{PUF}^{[5]}$.

弱 PUF 仅具有一个或少量的 CRP. 静态随机 存储器 PUF 是一种典型的弱 PUF 设计 ${ }^{[6]}$. 理想情 况下, 静态随机存储器单元在上电时默认取值为 比特 0 与比特 1 的概率相同; 但是由于工艺偏差的 影响，预期设计完全相同的晶体管会存在着包括 阈值电压等参数的不同, 使上电时静态随机存储 器单元的默认取值有了一定的倾向性. 其他的弱 PUF 还包括蝴蝶 $\mathrm{PUF}^{[6]}$ 等.

强 PUF 是指具有大量或指数级数量 CRP 的 PUF. 仲裁器 PUF 是一种典型的强 PUF 设计, 其利 用了具有相同设计的电路的时延差异，通过设置 激励的方式改变电信号传导路径, 从而产生大量
的 $\mathrm{CRP}^{[7]}$. 基于仲裁器 PUF 还有多种变型, 如异或 仲裁器 PUF ${ }^{[8]}$ 和模糊仲裁器 PUF ${ }^{[9]}$ 等. 其他的强 PUF 还包括环形振荡器(ring oscillator, RO) $P_{U}{ }^{[8]}$ 、 动态 DRAM PUF ${ }^{[10]}$ 、电流镜 $P U F^{[11]}$ 、可重构 RO PUF $^{[12]}$ 和混合 RO 仲裁 PUF $^{[13]}$ 等.

衡量 PUF 性能的好坏有多种指标, 其中, 均 匀性和独特性是相当重要的指标. 均匀性是指对 于同一个 PUF, 测试不同的激励, 其响应为比特 0 或比特 1 占全部响应的比例, 理想值为 $50 \%$. 独特 性是指对于具有相同设计的不同 PUF, 对其施加 相同的激励, 其响应为比特 0 或比特 1 占全部响应 的比例，理想值为 $50 \%$.

由于存在芯片固有时延、时延路径不对称等因 素, 传统 RO PUF 的均匀性和独特性都与理想值有 一定差异. 本文提出了可调可重构的 RO PUF 设计 方法来解决这一问题.

\section{2 传统 RO PUF}

传统 RO PUF ${ }^{[8]}$ 是一种典型的强 PUF 设计方 法, 其利用了具有相同设计的 RO 由于工艺偏差的 不同，导致环路振荡周期有所区别的特点，RO 结 构如图 1 所示.

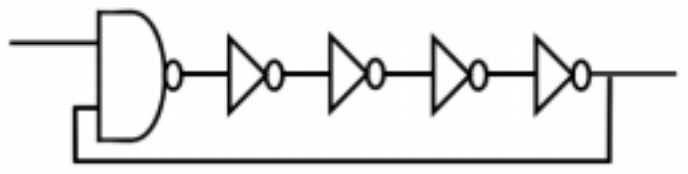

图 $1 \mathrm{RO}$ 结构

传统 RO PUF 结构如图 2 所示, 它由 2 个多路 选择器、 $N$ 个设计完全相同的 RO, 2 个计数器和 1 个比较器组成. 通过对多路选择器输入激励, 便会 有 2 个 $\mathrm{RO}(1$ 个 $\mathrm{RO}$ 对) 被选择, 2 个计数器分别反 映了其振荡频率. 计数器数值越大, 振荡频率越 高; 反之，频率越低. 使 2 个 RO 工作相同的时间, 通过比较器比较计数器的数值, 便可以获得响应.

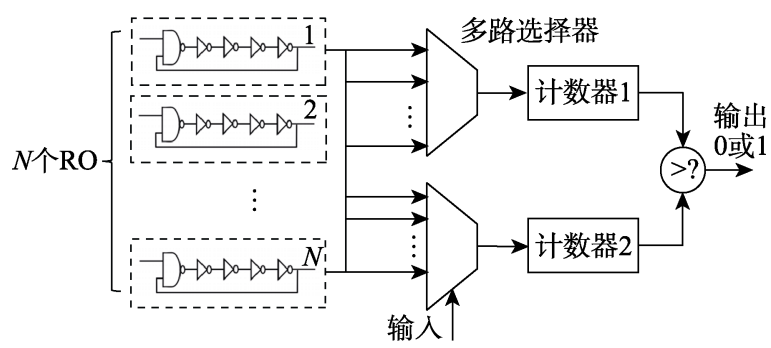

图 2 传统 RO PUF 结构 
文献[12]针对传统 RO PUF 做了独特性实验, 通过多种策略产生响应, 其中最好的独特性为 $45.51 \%$, 最差仅为 $23.17 \%$ ，与理想值仍存在一定 差距. 同时，在文献[14]针对传统 RO PUF 的均匀 性与独特性不够理想的问题, 提出了多种响应提 取策略, 并依次对所提出的种种提取策略进行实 验，最终得出最优的策略. 由于文献[14]的提取策 略仅是对芯片上位置相近的 RO 进行结合产生响 应，而不是在芯片上所有的 $\mathrm{RO}$ 中寻找适合的 $\mathrm{RO}$ 结合生成输出响应, 故 PUF 的均匀性和独特性虽 有所提升，但仍不是十分理想; 其中，效果最好的 提取策略的信息熵约为 0.46 .

\section{3 可重构 RO PUF}

在传统 RO PUF 的基础上，文献[12]提出了可 重构 RO PUF，其特点是将传统 RO PUF 的 $N$ 个振 荡器改为了 2 个可重构 $\mathrm{RO}$, 同时去掉了多路选择 器. 可重构 RO 和可重构 RO PUF 结构如图 3 所示.

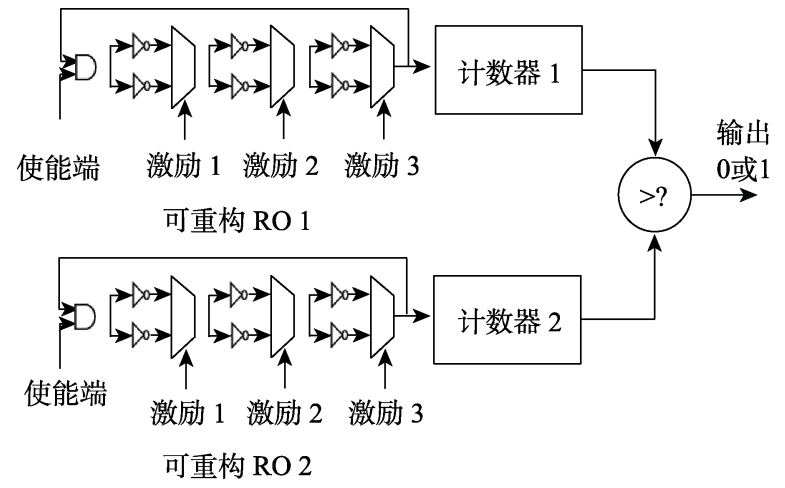

图 3 可重构 RO 和可重构 RO PUF 结构

\section{2 本文设计}

针对第 1 节提到的传统 RO PUF 的均匀性与独 特性不够理想的问题，本文提出了可调可重构的 RO PUF 设计. 以产生 7 比特响应的可调可重构 RO PUF 为例, 如图 4 所示, 该设计主要包括可重 构 RO 模块、整合器模块和裁决器模块. 这 3 个模

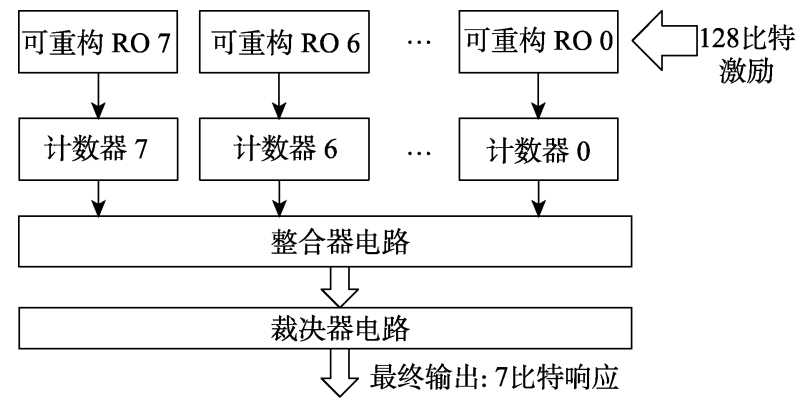

图 4 可调可重构 RO PUF 结构
块相互协作, 实现了保障 RO PUF 的均匀性与独特 性尽可能趋于理想值 50\%的目的.

\section{1 设计原理}

对 RO 环路时延进行建模，即

$$
\begin{gathered}
T_{\text {delay }}=T_{\text {expect }}+T_{\text {intrisic }}+T_{\text {variation }} \\
f=1 / T_{\text {delay }}
\end{gathered}
$$

其中, $T_{\text {delay }}$ 为环路总时延; $T_{\text {expect }}$ 为设计时预期振 荡延时，对于设计完全一致的 RO, $T_{\text {expect }}$ 相同; $T_{\text {variation }}$ 为由工艺偏差引起的环路时延差异, 是 PUF 正常工作的核心时延部分; $T_{\text {intrisic }}$ 为芯片固有 时延, 即使是同一块晶圆上的芯片, 仍存在诸如衬 底厚度不一致等现象，人为无法控制，文献[15]的 实验展示了 $T_{\text {intrisic }}$ 对 $\mathrm{RO}$ 频率的巨大影响.

对于传统 RO PUF，响应由

$$
R= \begin{cases}0, & \text { if } f_{1}>f_{2} \\ 1, & \text { otherwise }\end{cases}
$$

产生. 经过数学变换后, $R$ 可以表示为

$$
R= \begin{cases}0, & T_{\text {delay1 }}<T_{\text {delay2 }} \\ 1, & \text { otherwise }\end{cases}
$$

通过分析不同 RO 环路总时延的差值, 可确定 RO PUF 的响应, 即

$$
T_{\text {delay } 1}-T_{\text {delay } 2}=\Delta T_{\text {intrisic }}+\Delta T_{\text {variation }}
$$

其中, 当差值小于 0 时, 输出响应为比特 0 ; 否则 为比特 1

同时, 为了使 RO PUF 响应只与工艺偏差相 关, 使 RO PUF 均匀性和独特性趋于理想值, 需要 尽可能地减小 2 个 $\mathrm{RO}$ 的 $\Delta T_{\text {intrisic }}$ 的取值. 如图 5 所 示,横坐标代表 2 个位于同一块芯片上的且具有完 全相同设计的可重构 RO, 分别为可重构 RO 1 与可 重构 RO 2; 纵坐标代表振荡频率. 由于可重构 RO 存在多种配置情况，因此每个可重构 RO 对应的振 荡频率为一个区间，频率段 1 和频率段 2 分别代表 2 个可重构 RO 的振荡频率区间. 因为 2 个可重构 $\mathrm{RO}$ 在设计上是完全相同的, 所以两者的 $T_{\text {expect }}$ 是 完全一致的. 假设 2 个可重构 RO 所处的芯片位置 的特性(如衬底厚度等)有较大差别, 即 $T_{\text {intrisic }}$ 有较 大差别, 即由 $T_{\text {intrisic }}$ 影响的 2 个可重构 RO 的振荡 频率区间几乎没有重合部分, 会使由这 2 个 $\mathrm{RO}$ 组 成的可重构 RO PUF 产生的响应比特无均匀性与 独特性可言. 因此, 需要将 $T_{\text {intrisic }}$ 接近的可重构 $\mathrm{RO}$ 挑选并结合在一起产生响应比特, 以减小 $\Delta T_{\text {intrisic }}$ 的取值. $T_{\text {intrisic }}$ 直接影响计数器的数值, 设 计整合器模块将计数器值接近的可重构 $\mathrm{RO}$ 组合 
在一起, 可减弱 $\Delta T_{\text {intrisic }}$ 对响应比特均匀性和独特 性的不良影响. 同时, 需要设计裁决器模块对经整 合器模块处理后的数据进行裁决, 从而产生最终 的响应比特.

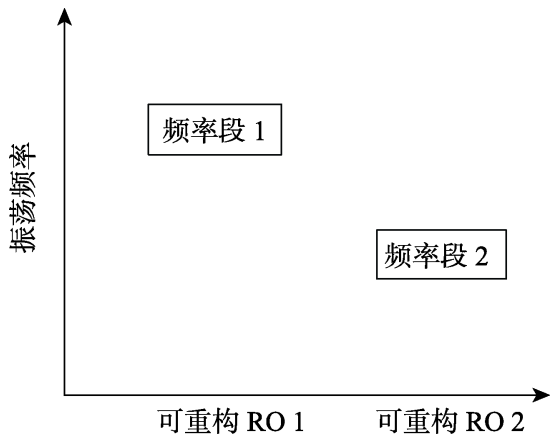

图 $5 T_{\text {intrisic }}$ 相差很大的 2 个可重构 $\mathrm{RO}$

\section{2 可重构 RO 模块}

可重构 RO 模块由多组设计完全一致的可重 构 RO-计数器组构成. 本文以产生 7 比特响应的设 计为例, 结构如图 6 所示.

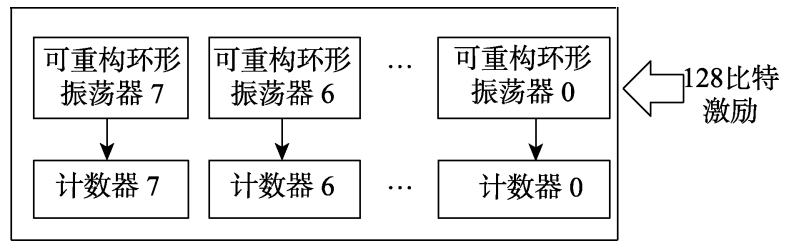

图 6 可重构 RO 模块

为了尽可能地节省芯片面积, $\mathrm{RO}$ 采用了如图 3 所示的可重构 RO. 同时, 为了产生数量尽可能 多的 $\mathrm{CRP}$, 将每一个可重构 $\mathrm{RO}$ 设计为 128 输人, 就可以实现最多 $2^{128}$ 个 CRP. 在电路工作时, 每一 个可重构 RO 均工作相同的时间，计数器数值反映 了其振荡频率差异.

\section{3 整合器模块}

整合器模块对可重构 RO 模块产生的计数器 数值进行处理. 整合器模块结构如图 7 所示, 它由 标号寄存器、排序电路、数值寄存器和减法器组成.

$T_{\text {intrisic }}$ 会对计数器数值产生较大影响, $T_{\text {intrisic }}$ 接近的 RO, 计数器数值也会更接近. 为了减弱 $T_{\text {intrisic }}$ 对 RO PUF 均匀性和独特性的影响, 在电路 工作之前, 预先根据计数器数值的大小, 通过输人 调整比特的方式预先为标号寄存器赋值. 例如, 对 数值最大的计数器相对应的标号寄存器赋值为 $3^{\prime} \mathrm{b} 111$, 对数值最小的计数器相对应的标号寄存 器赋值为 $3^{\prime} \mathrm{b} 000$, 其余的依次类推.

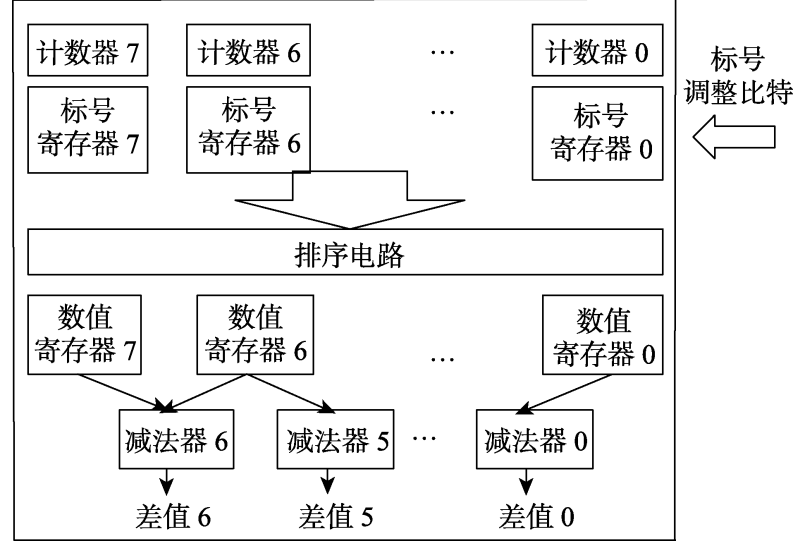

图 7 整合器模块

排序电路根据标号寄存器存储的标号, 将与 标号寄存器相对应的计数器数值按照标号的顺序 依次排列, 并将排列后的数值按照顺序存人数值 寄存器中, 排序电路的流程如图 8 所示.

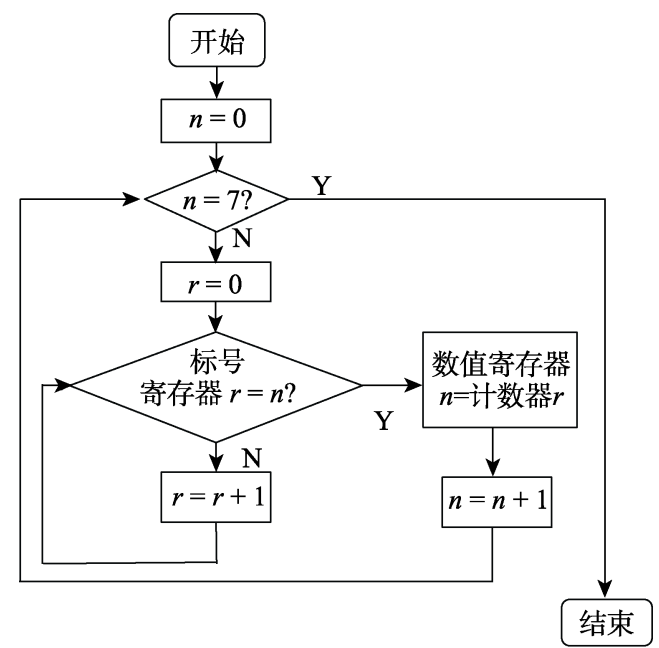

图 8 排序电路流程图

减法器将排序电路按照标号排序的计数器数 值两两作差. 经过排序处理, 2 个相邻的数值寄存 器受到 $T_{\text {intrisic }}$ 的影响更为相近, 通过减法运算就可 以使 $\Delta T_{\text {intrisic }}$ 尽可能小, 使 RO PUF 的均匀性与独 特性更加理想.

\section{4 裁决器模块}

裁决器模块的作用是将经整合器模块处理过 后的数据进行裁决, 产生最终的输出响应. 裁决器 模块的结构如图 9 所示, 它由阈值存储器和比较器 组成.

为了保证输出响应的均匀性, 需要在裁决响 应时输人阈值调整比特, 其设计方法为: 通过测试 一定数量的计数器值, 并经整合器模块处理后, 收 集产生的差值, 取各组差值的中位数作为阈值调 整比特. 


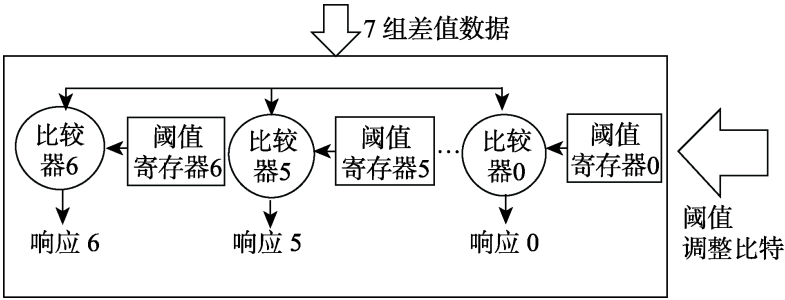

图 9 裁决器模块

根据中位数获得的國值调整比特与整合器模 块产生的差值, 用比较器进行比较：若差值小于國 值调整比特, 输出为比特 0 , 其余情况均输出比特 1. 依次比较, 便可以得出所有的输出响应.

\section{3 实验结果与分析}

为了验证所述设计方法的有效性，在 Xilinx Zynq-7000 Soc ZC702 FPGA 开发板上进行实验验 证. 其中, 可重构 RO 模块由 8 个相互独立且设计 完全相同的 128 比特可重构 RO-计数器组构成, 一 共产生 7 比特响应, 实现了 7 个 PUF. 其中, 每个 可重构 RO 由 385 个查找表(look up table, LUT)组 成, 8 个可重构 RO 共由 3080 个 LUT 组成, 实现了 7 个 PUF. 每当设计上多增加一个可重构 RO, 便会 多增加一个响应输出比特, 即多实现一个 PUF, 同 时其硬件开销也会增加 385 个 LUT.

实验分为 2 个阶段. 第 1 阶段，随机产生一定 数量的激励, 收集可重构 RO 模块中的计数器数 值, 并将通过获得的数据计算得出的标号调整比 特和阈值调整比特写人标号寄存器和阈值寄存器 中. 第 2 阶段, 在正常工作环境下，随机产生并输 人 $10^{5}$ 个激励, 收集产生的响应, 分析与计算改善 过的 RO PUF 均匀性和独特性的变化.

可调可重构 RO PUF 均匀性实验结果如表 1 所示.

表 1 可调可重构 RO PUF 均匀性实验结果 \%

\begin{tabular}{cc}
\hline PUF 序号 & 均匀性 \\
\hline 1 & 49.72 \\
2 & 49.48 \\
3 & 48.14 \\
4 & 49.22 \\
5 & 49.48 \\
6 & 49.88 \\
7 & 49.62 \\
\hline 平均值 & 49.36 \\
方差 & 0.30 \\
\hline
\end{tabular}

为了分析整合器模块对 RO PUF 独特性的影 响, 分别计算了以下 2 种情况的可调可重构 $\mathrm{RO}$ PUF 的独特性.

（1）移除整合器模块，直接将可重构 RO 模块 与裁决器模块相连.

(2) 不移除整合器模块, 仍采取如图 4 所示的 可调可重构 RO PUF 结构.

可调可重构 RO PUF 独特性实验结果如表 2 所示.

表 2 可调可重构 RO PUF 独特性实验结果 \%

\begin{tabular}{ccc}
\hline PUF 序号 & 无整合器 & 有整合器 \\
\hline 1 & 43.70 & 49.39 \\
2 & 36.52 & 44.83 \\
3 & 34.39 & 42.92 \\
4 & 36.26 & 47.59 \\
5 & 36.91 & 46.63 \\
6 & 42.88 & 49.63 \\
7 & 39.13 & 48.49 \\
\hline 平均值 & 38.54 & 47.07 \\
方差 & 10.70 & 5.20 \\
\hline
\end{tabular}

由表 1 可知, 在 7 组 PUF 中, PUF3 的均匀性 最差, 为 $48.14 \%$; PUF6 的均匀性最接近理想, 为 $49.88 \% .7$ 组 PUF 均匀性的平均值为 $49.36 \%$, 方差 为 0.30 .

由表 2 可知，在无整合器的条件下， 7 组 PUF 中, PUF3 的独特性最差, 为 $34.39 \%$; PUF1 的独特 性最接近理想, 为 $43.70 \% ; 7$ 组 PUF 独特性的平均 值为 $38.54 \%$, 方差为 10.70 . 在加有整合器的条件 下, 7 组 PUF 中, PUF3 的独特性最差, 为 $42.92 \%$; PUF6 的独特性最好, 为 $49.63 \% ; 7$ 组 PUF 独特性 的平均值为 $47.07 \%$, 方差为 5.20 . 含有整合器的 设计相对于无整合器的设计, 独特性均值提升了 $22.13 \%$, 方差降低了 $51.40 \%$, 从而验证了可调可 重构 RO PUF 的有效性.

相比文献[12,14]，本文方法在独特性方面有 所提升，更趋近于 $50 \%$ 的理想值，具体如表 3 所示.

表 3 与其他方法在独特性方面的对比 \%

\begin{tabular}{ll}
\hline 方法 & 独特性 \\
\hline 文献[12] & 最好 45.51 \\
文献[14] & 约为 46.00 \\
本文 & 47.07 \\
\hline
\end{tabular}

同时，本文方法从芯片中所有 RO 中选取产生 响应均匀性和独特性最为理想的组合产生输出响 
应，与其他工作仅从邻近 RO 中选取组合产生响应 相比，取得了更好的效果.

值得注意的是，本文调整比特的写人有多种 方法, 如可通过只读存储器写人调整比特来提升 PUF 的安全性.

\section{4 结 语}

在短短 20 年的发展历程中，PUF 已广泛应用 于多个领域，具有广阔的发展前景与应用空间. 针 对传统 RO PUF 均匀性与独特性不够理想的问题, 本文通过对 RO 振荡周期进行数学建模，找到了影 响其均匀性与独特性的原因, 并提出了可调可重 构的 RO PUF 的设计方法. 实验结果表明，可调可 重构的 RO PUF，其均匀性的平均值为 $49.36 \%$ ，独 特性的平均值为 $47.07 \%$, 均趋于 $50 \%$ 的理想值, 取得了一定的效果. 今后, 将围绕如何使 PUF 产 生稳定、可靠的响应比特进行研究.

\section{参考文献(References):}

[1] Rührmair U, Holcomb D E. PUFs at a glance[C] //Proceedings of the Conference on Design, Automation \& Test in Europe. Los Alamitos: IEEE Computer Society Press, 2014: Article No.347

[2] Negka L, Gketsios G, Anagnostopoulos N A, et al. Employing blockchain and physical unclonable functions for counterfeit IoT devices detection[C] //Proceedings of the International Conference on Omni-Layer Intelligent Systems. New York: ACM Press, 2019: 172-178

[3] Tuyls P, Batina L. RFID-tags for anti-counterfeiting[C] //Proceedings of the Cryptographers' Track at the RSA Conference on Topics in Cryptology. Heidelberg: Springer, 2006: $115-131$

[4] Che Wenjie. Research on hardware security techniques based on hardware metering and hardware watermarking[D]. Changsha: Hunan University, 2013(in Chinese)

(车文杰. 基于防伪控制与可验证水印的硬件安全技术研究 [D]. 长沙：湖南大学, 2013)

[5] Guajardo J, Kumar S S, Schrijen G J, et al. FPGA intrinsic
PUFs and their use for IP protection[C] //Proceedings of Cryptographic Hardware and Embedded Systems. Heidelberg: Springer, 2007: 63-80

[6] Kumar S S, Guajardo J, Maes R, et al. Extended abstract: the butterfly PUF protecting IP on every FPGA[C] //Proceedings of the IEEE International Workshop on Hardware-Oriented Security and Trust. Los Alamitos: IEEE Computer Society Press, 2008: 67-70

[7] Lim D, Lee J W, Gassend B, et al. Extracting secret keys from integrated circuits[J]. IEEE Transactions on Very Large Scale Integration Systems, 2005, 13(10): 1200-1205

[8] Suh G E, Devadas S. Physical unclonable functions for device authentication and secret key generation[C] //Proceedings of the 44th ACM/IEEE Design Automation Conference. Los Alamitos: IEEE Computer Society Press, 2007: 9-14

[9] Ye J, Hu Y, Li X W. OPUF: obfuscation logic based physical unclonable function[C] //Proceedings of the 21st IEEE International On-Line Testing Symposium. Los Alamitos: IEEE Computer Society Press, 2015: 156-161

[10] Xiong W J, Schaller A, Katzenbeisser S, et al. Dynamic physically unclonable functions[C] //Proceedings of the 2019 on Great Lakes Symposium on VLSI. New York: ACM Press, 2019: 311-314

[11] Kumar R, Burleson W. On design of a highly secure PUF based on non-linear current mirrors[C] //Proceedings of the IEEE International Symposium on Hardware-Oriented Security and Trust. Los Alamitos: IEEE Computer Society Press, 2014: 38-43

[12] Maiti A, Schaumont P. Improved ring oscillator PUF: an FPGA-friendly secure primitive[J]. Journal of Cryptology, 2011, 24(2): 375-397

[13] Yanambaka V P, Mohanty S P, Kougianos E, et al. PMsec: physical unclonable function-based robust and lightweight authentication in the Internet of medical things[J]. IEEE Transactions on Consumer Electronics, 2019, 65(3): 388-397

[14] Yu Yifei. The comparison strategies and reconfiguration of ring oscillator physical unclonable functions[D]. Nanjing: Nanjing University of Aeronautics and Astronautics, 2014(in Chinese) (余益飞. 环形振荡器物理不可克隆函数的比较策略及可重 构研究[D]. 南京: 南京航空航天大学, 2014)

[15] Wu Jin. Research and design of key technology of physical unclonable function based on ring oscillator[D]. Zhengzhou: Information Engineering University, 2018(in Chinese) (吴缙. 基于环形振荡器的物理不可克隆函数关键技术研究 与设计[D]. 郑州: 信息工程大学, 2018) 